\title{
Personal Decision Aiding: Some Observations about the Beach Birth-Planning Procedure
}

\author{
J. Frank Yates and William M. Goldstein \\ The University of Michigan
}

\begin{abstract}
The decision-aiding procedure developed by Beach and his colleagues for birth planning is reviewed. It is shown that the models underlying the Beach procedure have some unusual and problematic formal consequences. Contrary to the claims of Beach et al., the procedure cannot rely on a simple interpretation of standard utility theory for its justification. It is noted that, despite its formal difficulties, the Beach procedure has a unique feature for alerting the decision maker to potentially significant outcomes which could be adapted profitably in other decision-aiding settings besides birth planning. Suggestions for how to address the theoretical shortcomings of the Beach technique are offered.
\end{abstract}

Decision analytic procedures grounded in the basic notion of expected utility (EU) maximization (von Neumann \& Morgenstern, 1944; Savage, 1954) have become commonplace in many settings. Most applications of these decision aids have been restricted to such "public" arenas as business, engineering, medicine, and government (Schlaifer, 1969; Morris, 1977; Weinstein \& Fineberg, 1980). It seems that a primary reason for the restriction of their use to these domains is that these procedures are often difficult and expensive to apply. Decision analysts have implicitly or explicitly appropriately argued that such methods should be used only when the stakes, that is, the opportunity costs of making poor decisions, are high enough to warrant the great expenditure of resources required (e.g., Winkler, 1972, p. 220).

It is thus a challenging task to try to develop cost-effective versions of standard decision analytic tools that can be usefully applied to "small," personal decision problems. It appears that to date one of the most systematic attempts to construct, test, and implement a personal decisionaiding routine predicated on formal decision theoretic ideas is that made by Beach and his colleagues (Beach, Townes, Campbell, \& Keating, 1976;

It is our pleasure to acknowledge the useful discussions about the issues of this paper we have had with other members of the Human Performance Center Decision Group, Peter Politser, Stephen Eraker, and Keith Levi. We also appreciate the helpful comments of Lee Beach on an earlier draft of the paper. The opinions expressed in the paper are ours; the individuals mentioned above may not agree with all of those opinions. Requests for reprints should be addressed to J. Frank Yates, 136 Perry Building, Department of Psychology, University of Michigan, Ann Arbor, MI 48104. 
Wood, Campbell, Townes, \& Beach, 1977; Beach, Townes, \& Campbell, 1978a, 1978b; Beach, Campbell, \& Townes, 1979). As such, the Beach approach might be seen by some as a prototype for how one might go about adapting decision analytic methods to personal decision making. In addition, the Beach procedure assumes substantive significance in that it serves as a guide for counseling people about an especially important choice, whether or not to attempt to give birth to a child. ${ }^{1}$

This paper reviews the Beach procedure. It is shown that the models guiding the procedure lead to decision rules that are surprising and probably inappropriate. Beach et al. contend that these rules are instances of EU maximization. They have not demonstrated that this is so, however. We submit that the Beach decision rules might possibly maximize EU only under very restrictive and implausibly acceptable assumptions. Thus, the Beach procedure cannot rely upon utility theory as a rationale for its use. Despite its shortcomings, we note a feature of the Beach procedure that is unique and appealing and that might be incorporated to advantage in other decision analytic methods, in both personal and public settings. Finally, we offer some concrete suggestions for how Beach et al. might address the formal deficiencies of their technique and thereby build upon its current strengths.

\section{THE MODELS}

\section{The 1976 Model}

Beach et al. construct their procedure around what they call a "hierarchical utility model." As we shall show, two different models and attendant decision rules have been proposed, although it seems that Beach et al. consider the models to be equivalent. What we shall call the 1976 model appeared in that year in a scholarly journal account of the decision-aid procedure (Beach, Townes, Campbell, \& Keating, 1976). What we will call the 1978 model is implied in the instructions and clinician's manual for an operational version of the procedure published by the National Alliance for Optional Parenthood (Beach, Townes, \& Campbell, 1978a, 1978b). We begin the discussion with the 1976 model.

It is easiest to understand the 1976 model if it is described both schematically and algebraically. Figure 1 is an adaptation of Beach et al.'s (1976) Fig. 1. To make various distinctions as clear as possible, whereas Beach et al. used only one hierarchical schematic, we have drawn two, one for the Birth (B) option, another for the No Birth (NB) option. Also, we use slightly different, more general notation. In the illustration of the formalisms of their model presented in their Fig. 1, Beach et al. employ a

\footnotetext{
${ }^{1}$ We refer to the "Beach" procedure for simplicity of reference; no slighting of Beach's colleagues is intended.
} 


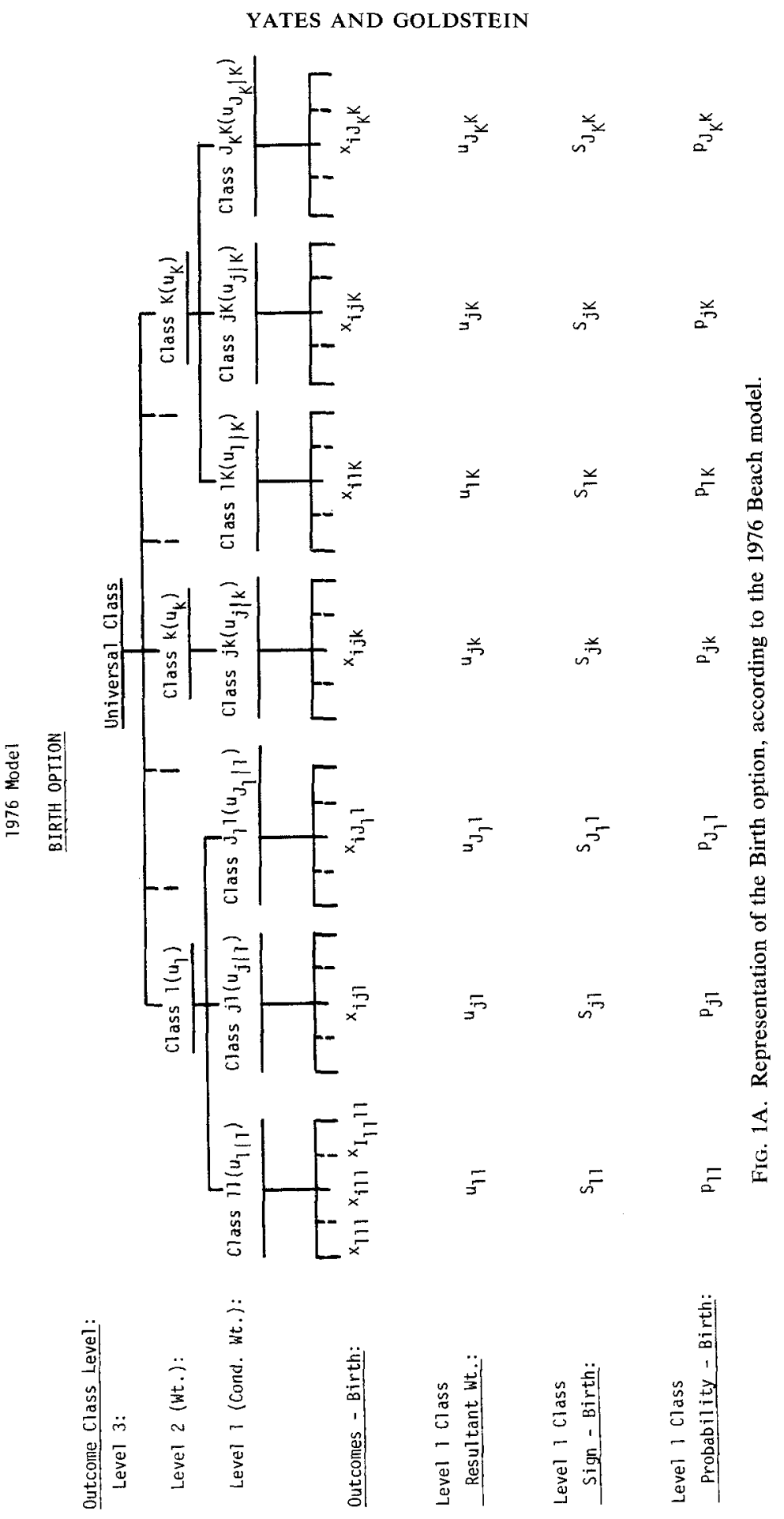




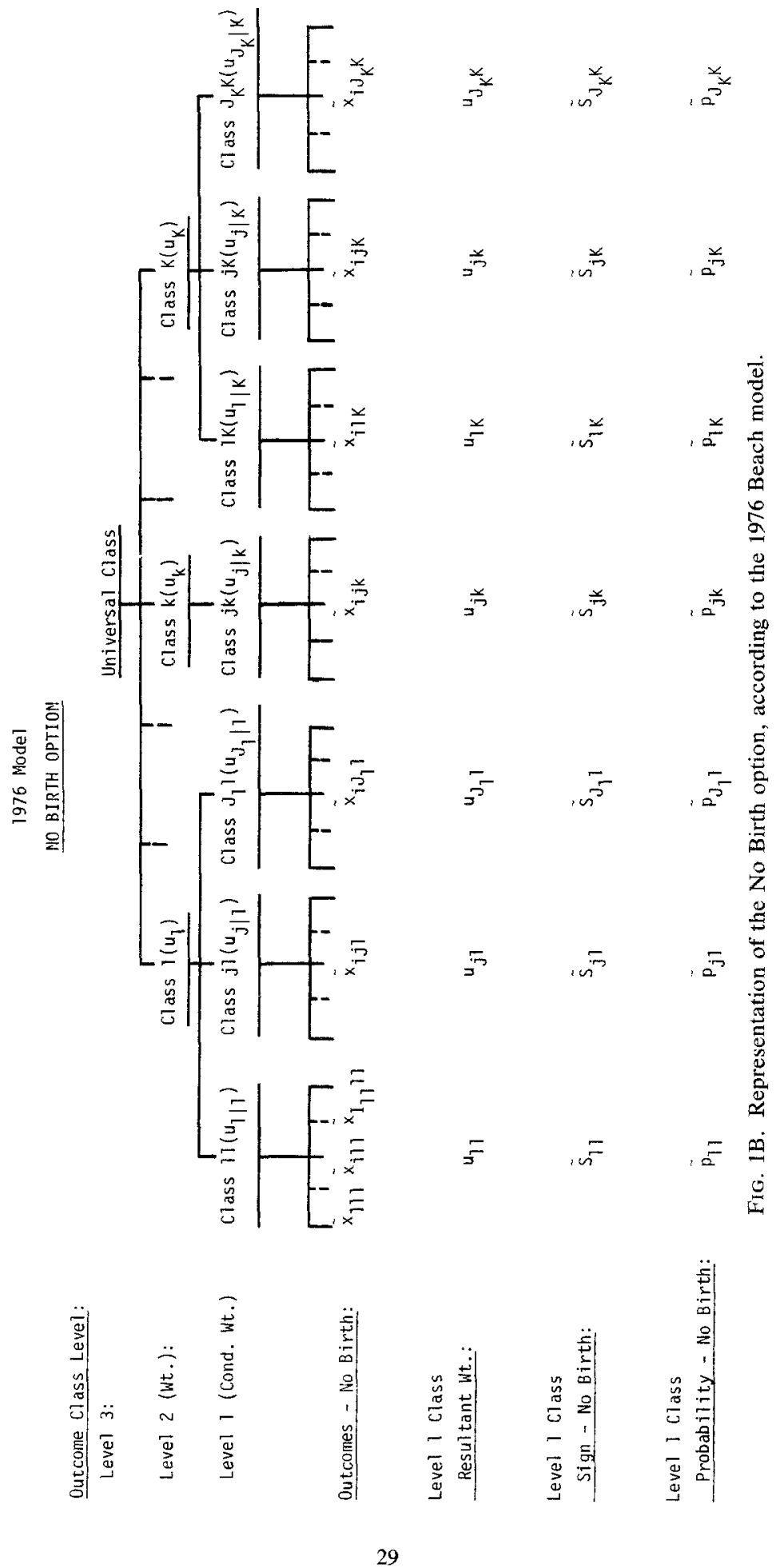


hierarchy which extends to three levels. Their discussion and empirical procedures, however, make use of a four-level hierarchy. Since it simplifies the discussion and represents no loss of generality, we describe the 1976 Beach model in terms of a three-level hierarchy.

For the moment, focus on the Birth option, Fig. 1A. Let us first examine the basic elements of the model. Consider the "Outcomes-Birth" section of the figure. The $x_{i j k}$ represent various individual outcomes or outcome categories which might result from having a child. It is assumed that the $x_{i j k}$ are partitioned into mutually exclusive, exhaustive, and "independent"' classes at what we call here Level 1 . For example, $x_{111}$ might be "Child might balance the number of boys and girls in our family" and the Level 1 class to which $x_{111}$ belongs (Class 11) might be "Family size and sexes of children." It is further assumed that Level 1 classes are similarly partitioned into mutually exclusive, exhaustive, and independent Level 2 classes. For instance, "Family size and sexes of children" might be subsumed under Level 2 Class 1, "Family characteristics." The hierarchy ends at the level consisting of only the single "Universal Class" of all outcomes that might occur.

Importance ratings apply to each level of the hierarchy. Beach et al. (1976, p. 101, Fig. 1) call these ratings "utilities." For generality and simplicity, we merely call them "weights." Level 2 weights $u_{k}$ are constrained to be between 0 and 1 and must sum to 1 across all Level 2 outcome classes. These weights are understood to reflect the relative importance of the respective classes for the decision maker's decision. Level 1 "conditional weights" $u_{j \mid k}$ are similarly constrained and interpreted within a given Level 2 class. That is, each of the weights for Level 1 classes subsumed under Level 2 Class $k$ are required to be between 0 and 1 and must sum to 1 . They should also reflect the relative importance of their respective Level 1 classes for the Level 2 class to which they belong. Beach et al. define a quantity which we call the "resultant weight" $u_{j k}$ for each Level 1 class. Resultant weights are obtained by a simple multiplication rule using Level 2 weights and Level 1 conditional weights: $u_{j k}=u_{k} \cdot u_{j \mid k}$.

Beach et al. associate a $\pm \operatorname{sign} S_{j k}$ with each of the Level 1 classes. A particular class receives a positive or negative sign, respectively, if the class as a whole seems to constitute "an argument in favor of or an argument against the decision to have a (another) child" (Beach et al., 1976, p. 106).

The final key ingredient of the model consists of Level 1 class probabilities $p_{j k}$. For Class $j k, p_{j k}$ represents the decision maker's "subjective

\footnotetext{
${ }^{2}$ The particular sense in which the term "independent" is used is not specified by Beach et al. $(1976$, p. 101).
} 
probability ... that the outcomes that define that class would, in fact, accrue to him were he to elect [to have a child]" (Beach et al., 1976, p. 102). It should be noted that distinctions are not made among the occurrences of all the individual outcomes $x_{i j k}$ within Class $j k$. That is, $p_{j k}$ is something of a broadly defined joint probability for an entire collection of events.

Now consider the No Birth option, represented by Fig. 1B. The basic hierarchy and weights for the No Birth option are identical to those for the Birth option. So let us consider Beach et al.'s assertions as to what differs between the two options. Of course, the specific, individual outcomes of the options are not the same. This is where the first important simplifying assumption is made. Each outcome $x_{i j k}$ resulting from the No Birth option is in a particular sense seen to be complementary or symmetric to one resulting from the Birth option: "that outcomes that would be gains if one were to have the child can be seen as losses if one were to not do so, and vice versa" (Beach et al., 1976, p. 102). The sign for Class $j k$ in the No Birth option is set to be opposite that for Class $j k$ in the Birth option: $\tilde{S}_{j k}=$ $-S_{j k}$. The final difference between the Birth and No Birth representations concerns probabilities. It is assumed that the Level 1 class probabilities for the options are complementary. That is, the No Birth option probability that Class $j k$ outcomes will occur is given by $\tilde{p}_{j k}=1-p_{j k}$.

How are all the various aspects of the representations combined to prescribe a decision? Beach et al. (1976) define what they refer to as a "subjective expected utility" (SEU) for each option and then take the difference. If the difference is positive, one decision is indicated; if the difference is negative, the opposite decision is advised. Further, "the larger this SEU is relative to the smaller one, the more clear cut the decision" (Beach et al., 1976, p. 102).

There are a number of difficulties with the assumptions and operations described. Some of these problems are rather general and their discussion is deferred to a later section of the paper on "Strengths and Weaknesses of the Procedure." Perhaps the most serious problem with the 1976 model becomes apparent when one writes out the above SEU prescription formally.

SEU for the Birth option is defined by Beach et al. (1976) as

$$
\operatorname{SEU(B)})_{1976}=\sum_{k=1}^{K} \sum_{j=1}^{J_{k}} p_{j k} S_{j k} u_{j k} \text {. }
$$

Similarly, SEU for the No Birth option is given by

$$
\operatorname{SEU}(\mathrm{NB})_{1976}=\sum_{k=1}^{K} \sum_{j=1}^{J_{k}} \tilde{p}_{j k} \tilde{S}_{j k} u_{j k} .
$$


This implies, of course, that the SEU difference in favor of having a child can be expressed as

$$
\Delta \mathrm{SEU}(\mathrm{B})_{1976}=\mathrm{SEU}(\mathrm{B})_{1976}-\mathrm{SEU}(\mathrm{NB})_{1976} .
$$

Let us reduce $\Delta \operatorname{SEU}(B)_{1976}$ :

$$
\begin{aligned}
\Delta \operatorname{SEU}(\mathrm{B})_{1976} & =\sum_{k=1}^{K} \sum_{j=1}^{J_{k}}\left[p_{j k} S_{j k}-\tilde{p}_{j k} \tilde{S}_{j k}\right] u_{j k} \\
& =\sum_{k=1}^{K} \sum_{j=1}^{J_{k}}\left[p_{j k} S_{j k}-\left(1-p_{j k}\right)\left(-S_{j k}\right)\right] u_{j k} \\
& =\sum_{k=1}^{K} \sum_{j=1}^{J_{k}}\left[p_{j k} S_{j k}+S_{j k}-p_{j k} S_{j k}\right] u_{j k} \\
\Delta \operatorname{SEU}(\mathrm{B})_{1976} & =\sum_{k=1}^{K} \sum_{j=1}^{J_{k}} S_{j k} u_{j k} .
\end{aligned}
$$

That Eq. (4) is an accurate representation of the Beach 1976 model can be verified by applying it to the hypothetical example provided by Beach $e t$ al. (1976) in their Fig. 1.

The most striking, and perhaps surprising, thing about Eq. (4) is that it contains no probabilities. Were it not for the fact that the sign indicators $S_{j k}$ in Eq. (4) can differ from one another, $\Delta \mathrm{SEU}(\mathrm{B})_{1976}$ would be identically +1 or -1 , since

$$
\begin{aligned}
\sum_{k=1}^{K} \sum_{j=1}^{J_{k}} u_{j k} & =\sum_{k=1}^{K} \sum_{j=1}^{J_{k}} u_{k} \cdot u_{j \mid k} \\
& =\sum_{k=1}^{K} u_{k} \sum_{j=1}^{J_{k}} u_{j \mid k} \\
& =\sum_{k=1}^{K} u_{k} \\
\sum_{k=1}^{K} \sum_{j=1}^{J_{k}} u_{j k} & =1,
\end{aligned}
$$

by the unit additivity assumption.

Of course, the reasons for the probabilities dropping out of the SEU difference are the probability complementarity assumption, i.e., $\tilde{p}_{j k}=1-$ 
$p_{j k}$, and the sign symmetry assumption, i.e., $\tilde{S}_{j k}=-S_{j k}$. The probability complementarity assumption is clearly unreasonable. Beach et al.'s (1976) rationale for the assumption is rather sketchy. After defining the subjective probability for a class in the Birth option, they merely say, "this also determines the probability, $1-p$, for [the No Birth option]" (p. 102). What Beach et al. seem to be saying with the complementarity assumption is that, for events $\mathrm{O} \equiv$ "outcome" and $\mathrm{C} \equiv$ "Child," $P(O \mid \vec{C})$ $=1-P(O \mid C)$. As a moment's reflection forces one to realize, on purely formal grounds, there is no necessary relation whatsoever between probabilities of events that are conditioned on disjoint prior events.

In substantive terms, what Eq. (4) indicates is that the subjective probability elicitation phase of the 1976 procedure is without purpose; the probabilities are never used. Obviously, this cannot have been intended by Beach et al. Equation (4) also implies that, to the extent that the 1976 model is predictive of actual behavior, e.g., birth choices, it does so purely on the basis of outcome class weights and signs. The certainty or uncertainty the person feels about the actual occurrence of those outcomes does not enter the picture at all.

\section{The 1978 Model}

It is considerably more difficult to reconstruct the 1978 model than the 1976 model. This is because the original description of the former model (Beach, Townes, \& Campbell, 1978a, 1978b) is presented in the form of response and scoring instructions for an "Optional Parenthood Questionnaire," rather than as an explicit theoretical exposition. Nevertheless, we believe the following is, in fact, an accurate rendering of Beach et al.'s developments. As in the case of the 1976 model, we have chosen notation that is different and more general than that used by the original authors.

Figure 2 presents a schematic representation of the Birth option according to the 1978 model. The notational conventions are essentially the same as those used for our description of the 1976 model. In terms of the basic constructs employed, there is only one major difference between the 1976 and 1978 models. Specifically, the assignment of weights, outcome signs, and probabilities starts one level lower in the hierarchy in the 1978 as compared to the 1976 model. Nevertheless, the weights, signs, and probabilities have the same basic interpretations and properties. Thus, the resultant weight for outcome $x_{i j k}$ is again given by a successive multiplication rule: $u_{i j k}=u_{i \mid j k} \cdot u_{j \mid k} \cdot u_{k}$. Also, unit additivity is still assumed: 

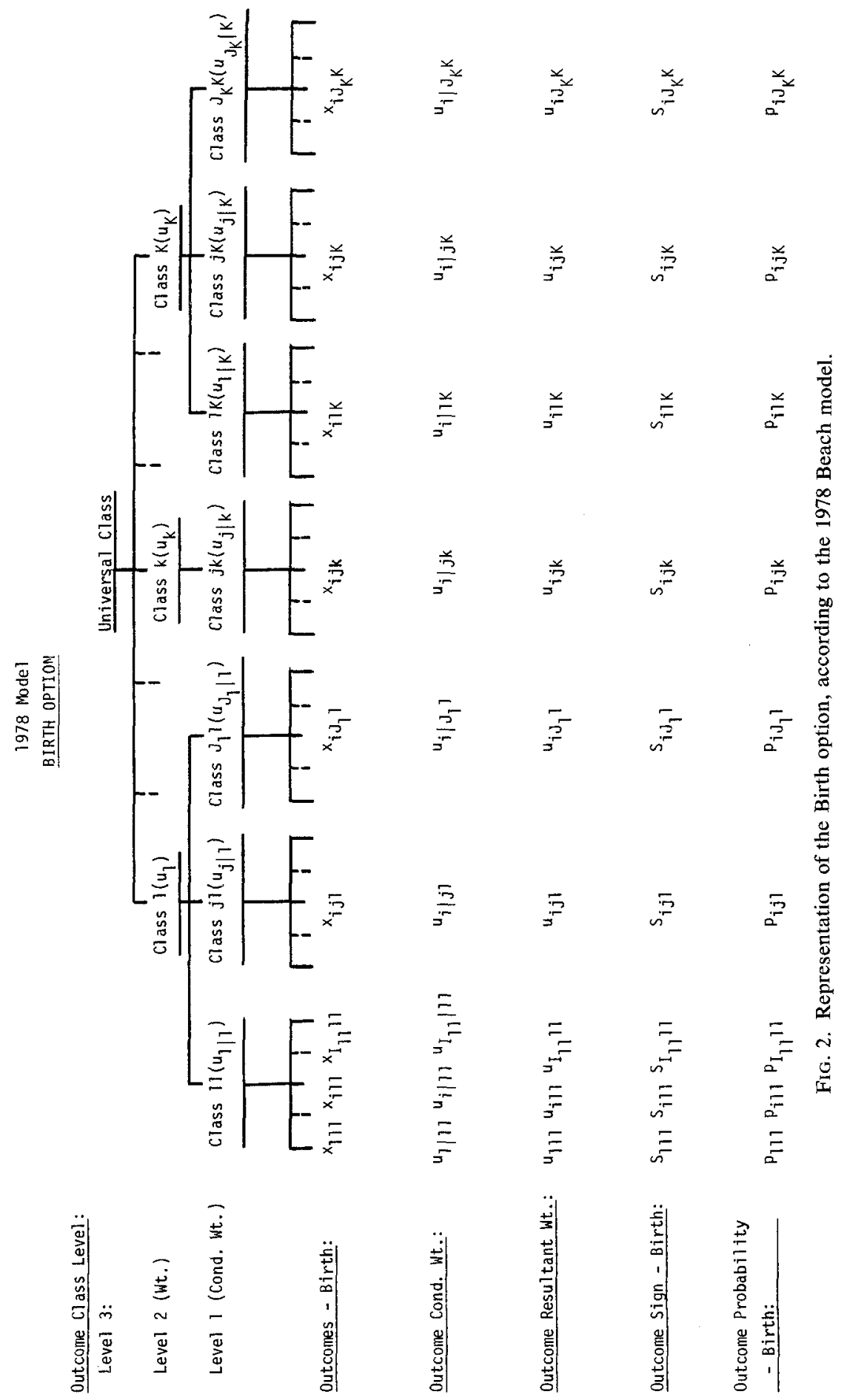


$$
\begin{aligned}
\sum_{k=1}^{K} \sum_{j=1}^{J_{k}} \sum_{i=1}^{I_{j k}} u_{i j k} & =\sum_{k=1}^{K} \sum_{j=1}^{J_{k}} \sum_{i=1}^{I_{j k}} u_{i \mid j k} \cdot u_{j \mid k} \cdot u_{k} \\
& =\sum_{k=1}^{K} \sum_{j=1}^{J_{k}} u_{j \mid k} \cdot u_{k} \sum_{i=1}^{I_{j k}} u_{i \mid j k} \\
& =\sum_{k=1}^{K} u_{k} \sum_{j=1}^{J_{k}} u_{j \mid k} \\
& =\sum_{k=1}^{K} u_{k} \\
\sum_{k=1}^{K} \sum_{j=1}^{J_{k}} \sum_{i=1}^{I_{j k}} u_{i j k} & =1 .
\end{aligned}
$$

It might be observed that, while the 1978 model permits outcome conditional weights $u_{i \mid j k}$ to be either 0 or 1 , it does not allow Level 1 conditional weights or Level 2 weights to take on those values. Substantively, what this means is that the decision maker is permitted to indicate irrelevance or overriding importance of lower-level, but not upper-level outcome classes. One should perhaps also note that (presumably to reduce computational complexity) the 1978 model restricts the probabilities the decision maker might express to three values, $.1, .5$, and .9 .

In terms of the rules for combining weights, signs, and probabilities, the differences between the 1976 and 1978 models are subtle but, as it turns out, very consequential. We have not constructed a schematic of the No Birth option hierarchy according to the 1978 model, in part because it is not clear exactly what such a representation would look like. We can, however, identify how various model components are combined for both the options. Because the instructions in the Beach et al. (1978a, 1978b) sources are strictly procedural, indicating no rationale, we cannot say exactly what the justification for the combination rules is.

The combination rules for the 1978 model turn on a partitioning of the Birth outcomes according to the signs the decision maker attaches to them. Recall that $S_{i j k}=+1$ if, for the given issue, the decision maker's "feelings and thoughts are mostly in favor of having a child" (Beach et al., 1978a, p. 3). $S_{i j k}=-1$ if those feelings and thoughts are against having a child. Let us define two index sets

$$
A+=\left\{(i, j, k) \mid S_{i j k}=+1\right\} \quad \text { and } \quad A-=\left\{(i, j, k) \mid S_{i j k}=-1\right\} .
$$

That is, $A+$ identifies those outcomes that orient the decision maker toward having a child; $A$-identifies those that orient the decision maker 
away from having a child. Also, recall that $p_{i j k}$ is the decision maker's subjective probability that having a child would result in outcome $x_{i j k}$ occurring.

In their procedural exposition, Beach et al. (1978a, 1978b) do not explicitly use the term "subjective expected utility (SEU)." To call their summary measures something and to be consistent with their earlier usage, we will call those summary measures SEUs. According to the 1978 model, the SEU for having a baby is given by

$$
\operatorname{SEU}(\mathrm{B})_{1978}=\sum_{A+} p_{i j k} u_{i j k}+\sum_{A-}\left(1-p_{i j k}\right) u_{i j k} \text {. }
$$

The SEU for not having a baby can be written as

$$
\operatorname{SEU}(\mathrm{NB})_{1978}=\sum_{A-} p_{i j k} u_{i j k}+\sum_{A+}\left(1-p_{i j k}\right) u_{i j k} .
$$

In Eqs. (7) and (8), the symbols $\sum_{A+}$ and $\sum_{A-}$ indicate that summations should be taken over all index ensembles in $A+$ and $A$-, respectively. Perhaps the most immediately obvious difference between the SEUs for the 1976 and 1978 models is that there are no negative signs in those of the latter model. Probabilities are also handled somewhat differently in the 1976 and 1978 models. The significant fact that should be kept in mind, however, is that the 1978 procedure still requires the decision maker to explicitly make probability statements conditional upon only one of the possible options, Birth.

The decision as to whether the decision maker should or should not have a child is again indicated by an SEU difference:

$$
\Delta \operatorname{SEU}(B)_{1978}=\operatorname{SEU}(B)_{1978}-\operatorname{SEU}(N B)_{1978},
$$

which reduces to

$$
\Delta \mathrm{SEU}(\mathrm{B})_{1978}=\sum_{A+}\left(2 p_{i j k}-1\right) u_{i j k}-\sum_{A-}\left(2 p_{i j k}-1\right) u_{i j k} .
$$

The decision rule is as follows. If $\triangle \mathrm{SEU}(\mathrm{B})_{1978}$ is greater than zero, having a child is indicated; if $\Delta$ SEU(B $)_{1978}$ is less than zero, not having a child is indicated.

Although Eq. (9) has a rather odd form, it has a certain heuristic appeal. It seems to represent a tradeoff between outcomes favoring and disfavoring having a child. Moreover, in contrast to the corresponding expression for the 1976 model, it does incorporate probabilities.

The previous analyses indicate that the 1976 and 1978 Beach models and decision rules are not identical. On closer inspection, it became apparent to us, however, that there is a very clearcut relationship between the SEUs yielded by the two models. Equation (9) can be rewritten as 


$$
\begin{aligned}
\Delta \mathrm{SEU}(\mathrm{B})_{1978} & =2 \sum_{A+} p_{i j k} u_{i j k}-\sum_{A+} u_{i j k}-2 \sum_{A-} p_{i j k} u_{i j k}+\sum_{A-} u_{i j k} \\
& =2 \sum_{A+} p_{i j k} S_{i j k} u_{i j k}+2 \sum_{A-} p_{i j k} S_{i j k} u_{i j k}-\left(\sum_{A+} u_{i j k}-\sum_{A-} u_{i j k}\right) \\
& =2 \sum_{k=1}^{K} \sum_{j=1}^{J_{k}} \sum_{i=1}^{I_{j k}} p_{i j k} S_{i j k} u_{i j k}-\left(\sum_{A+} S_{i j k} u_{i j k}+\sum_{A-} S_{i j k} u_{i j k}\right) \\
& =2 \sum_{k=1}^{K} \sum_{j=1}^{J_{k}} \sum_{i=1}^{I_{j k}} p_{i j k} S_{i j k} u_{i j k}-\sum_{k=1}^{K} \sum_{j=1}^{J_{k}} \sum_{i=1}^{I_{j k}} S_{i j k} u_{i j k}
\end{aligned}
$$

$$
\Delta \mathrm{SEU}(\mathrm{B})_{1978}=2 \mathrm{SEU}(\mathrm{B})_{1976}-\Delta \mathrm{SEU}(\mathrm{B})_{1976},
$$

from Eqs. (1) and (4), as generalized to the extended hierarchy. Making use of Eq. (3), the following result emerges:

$$
\Delta \mathrm{SEU}(\mathrm{B})_{1978}=\mathrm{SEU}(\mathrm{B})_{1976}+\mathrm{SEU}(\mathrm{NB})_{1976} .
$$

\section{STRENGTHS AND WEAKNESSES OF THE PROCEDURE}

Why should a prospective parent choose between having and not having a child according to the prescriptions of either the 1976 or 1978 Beach model? Ideally, one would like to be able to cite a statistically reliable track record for a decision-aiding procedure indicating that people who have followed the procedure have made decisions whose outcomes, on average, have been demonstrably more satisfying than the outcomes of decisions made by people who have not followed the procedure. It does not appear that anyone has yet demonstrated such "proof of the pudding" for any EU decision analytic procedure (cf. Selvidge, Note 1; Weinstein \& Fineberg, 1980). Beach et al. certainly do not present such evidence for their procedure.

Instead of relying upon demonstrations of the terminal efficacy of their methods, decision analysts typically justify the use of those methods by arguing that the details of the procedures are structurally advantageous (e.g., Howard, 1973). This is the tack taken by Beach et al., too. The rationales Beach et al. offer for their procedure seem to fall into three categories. First, they justify their approach on the basis of "Mathematical Decision Theory." Presumably, they mean subjective expected utility theory interpreted as a normative model, since they go on to say: "Decision Theory prescribes how these evaluations are to be summarized to yield an expectation for each course of action: The course of action with the most favorable expectation is the one that should be selected" (Beach et al., 1978b, p. 4). Second, Beach et al. rely on the decomposition princi- 
ple (Winkler \& Murphy, 1973), arguing that human attentional and other information processing limitations preclude the possibility that, left to their own devices, people can adequately perform the complex operations that seem to be required for expectation maximization. Finally, Beach $e t$ al. (1976) argue that their decision scheme should assist decision makers to "more clearly know their own thinking" (p. 100) about the various aspects of a birth decision problem. We will comment on each of these rationales in turn.

It is very apparent that Beach et al. consider the utility theory rationale to be the most fundamental justification for their decision-aiding routine. We submit that, unfortunately, their procedure cannot appeal to formal utility theory directly for its sensibleness. There are two primary reasons for this. The first is that, as the above analyses suggest, unless one makes very restrictive (and partly unspecified) assumptions, neither the 1976 nor the 1978 model is a version of subjective expected utility theory as it is defined in formal treatments (e.g., Savage, 1954; DeGroot, 1970; Keeney \& Raiffa, 1976). The second reason is that the Beach technique does not involve utility assessment methods which provide any assurance that the numbers attached to outcomes have the essential property of utilities, namely that expectations defined over utilities preserve preference orderings of alternatives which have uncertain consequences. In decision analytic procedures more closely tied to the axiomatic foundations of utility theory, a good deal of attention is devoted to consistency checks which amount to verification of the applicability of the theory's assumptions in the given situation and of the adequacy of the measures derived.

Hershey, Kunreuther, and Schoemaker (in press) have garnered evidence that formally defensible and equivalent, but operationally different, techniques for eliciting utility functions for money result in systematically different assessments. The Beach measurement techniques differ markedly from the standard techniques. Thus, it should be expected that the results yielded by the Beach methods will differ from those produced by the traditional methods. And there is no reason to anticipate that the difference will necessarily represent superiority for the Beach approach. The aspect of the Beach measurement method that is most troubling is that the client is asked to provide ratings under the (implicit) assumption of certainty, whereas the decision to have a child is a risky decision. Thus, in contrast to the presumption in standard utility function assessment techniques, there is little reason to believe that those ratings will embody the client's attitude toward risk (cf. Pratt, 1964). Some authors (e.g., Keeney \& Raiffa, 1976) have implied that it would be useful to reserve the term "value function" for functions expressing the worth of outcomes under certainty. The term "utility function" would then be used only to refer to functions indicating worth under conditions of uncertainty. The 
Beach technique seems to yield a value function rather than a utility function, according to these conventions. A utility function is necessarily also a value function, but not conversely.

Another discrepancy between formal utility theory and the Beach procedure is worth noting, too. Formal utility theory attaches no significance to the magnitude of the difference $\triangle \mathrm{SEU}(\mathrm{B})=\mathrm{SEU}(\mathrm{B})-\mathrm{SEU}(\mathrm{NB})$, other than its sign. Beach et al., as will be recalled, suggest that the larger $\triangle \mathrm{SEU}(\mathrm{B})$ is, the more strongly the Birth option is indicated. It should be borne in mind, however, that axiomatic treatments of utility theory do not take error of measurement into account and that the aim of such treatments is to rationalize simply choice of options, not strength of choice, whatever that might mean. If one assumes-and probably rightfully so- that all utility assignments have error associated with them, the magnitude of the assessed value of $\triangle \mathrm{SEU}(\mathrm{B})$ does have some importance. Specifically, the larger the assessed value of $\triangle S E U(B)$ is, the more assured one ought to feel that the "true" value of $\triangle S E U(B)$ is positive and, hence, according to a proper interpretation of utility theory, the Birth option is indicated.

The second justification for the Beach procedure, stressing the usefulness of breaking the decision problem into manageable chunks and combining judgments mechanically, seems reasonable. Nevertheless, as Winkler and Murphy (1973) point out, the "divide-and-conquer" expectation implicit in the decomposition strategy might not be realistic if the decision maker is not accustomed to making the kinds of judgments the strategy demands.

One of the major strengths of the Beach procedure is related to the third justification offered by Beach et al., the clarification of the decision maker's own thinking. By directly confronting the prospective parent with various issues that are likely to arise in parenthood, the procedure ought to enhance the person's chances of discovering his or her true feelings about those issues. When both prospective parents carry out and discuss the results of the procedure, it should be especially valuable. It should facilitate the identification and resolution of the partners' disagreements in terms of values and expectations. Such an effect would be reminiscent of Gardiner and Edwards' (1975) suggestion that multiattribute utility analysis can often be expected to help settle conflicts about public policy issues.

The formal aspects of standard decision analytic procedures, e.g., probability theory and utility theory, are concerned solely with "internal consistency," making certain that the decision maker's judgments and choices do not contradict one another. Much less attention is devoted to what Yates and Carlson (Note 2) refer to as the "external correspondence" of the person's decision processes. That is, few mechanisms 
are built into the procedures to assist the decision maker in making certain of the accuracy of his or her perceptions of the decision situation, including his or her own preferences. An analogy with symbolic logic exercises is apt. As long as a syllogism violates none of the rules of logic, the argument represented by the syllogism is valid, i.e., internally consistent. However, testing the argument against those rules cannot assure that the conclusions which are drawn make sense in the real world if the terms going into the argument have no firm basis in reality to start with, i.e., no external correspondence.

It is in respect to external correspondence that the Beach procedure seems to have its greatest potential. Left to their own powers of imagination, prospective parents, particularly first-time parents, probably overlook a large number of the aspects of parenthood and nonparenthood which would ultimately be significant to them-positively and negatively. So, even if impeccably internally consistent reasoning were applied to those prospective parents' judgments, their decisions might easily result in outcomes that are not only surprising, but disappointing. The operationalization of the Beach procedure serves to reduce the chance of such poor external correspondence.

The Beach models themselves are indifferent to the source of the list of outcomes to which the prospective parent attaches utilities and probabilities. In actual practice, however, the client is required to make judgments about a predetermined set of outcome classes. The outcome classes were not generated in a vacuum. Rather, they represent categories of consequences of parenthood and nonparenthood systematically solicited by Beach et al. (1976) from married couples who have thought hard about the issues. In fact, it might be surmised that Beach et al.'s respondents have actually experienced many of those consequences and found that they mattered to them. Thus, those consequences might also be expected to matter to many decision analysis clients using the Beach procedure who are contemplating having children. Analogous "alerting" to potentially significant decision consequences should prove valuable in almost any decision analytic situation, including traditional business and engineering ones.

\section{SUGGESTIONS}

There is nothing about Eqs. (4) and (9) themselves or even the standard EU expression, for that matter, which should compel the decision maker to say, "I obviously ought to make choices according to that formula!" The prescriptive force behind expected utility theory is embodied in the axioms that are necessary for representing a preference structure as EU maximization (von Neumann \& Morgenstern, 1944; Herstein \& Milnor, 1953; Savage, 1954; Luce \& Raiffa, 1957). Many people consider those 
axioms to be important, desirable principles of rationality. So, the primary reason a decision analysis client should be willing to permit his or her choices to be dictated by EU maximization is that, by so doing, he or she eliminates the possibility that any of those principles will be transgressed. Ideally, then, the decision analyst should always present the axioms to the client and see if he or she agrees (perhaps with a bit of persuasion) that one should try to avoid violating those axioms. If the client does agree, then the decision analysis should proceed. If not. . .

We have seen that neither of the Beach models is clearly a version of subjective expected utility (SEU) theory as it is commonly understood. With regard to the formalisms of their procedure, Beach et al. thus have three options. First, if one or both of their models $i s$ a restrictive, special case of SEU theory, they should demonstrate that this is so, making explicit what all the assumptions are. A client using their procedure should be permitted to indicate whether those restrictions represent principles he or she would like to preserve, along with the other SEU axioms. Second, if the models are not special cases of SEU theory, but Beach et al. still feel that their decision rules are reasonable, they should axiomatize their models (Krantz, Luce, Suppes, \& Tversky, 1971). Then clients should be confronted with the necessary axioms of the models and permitted to indicate whether they accept those axioms and thus agree with Beach et al. that the Beach decision rules are appropriate guides for their actions. Finally, Beach et al. might choose to modify their models so that they better conform to standard SEU theory. At the risk of being unnecessarily explicit, we briefly outline below what the application of the SEU model to a birth planning decision would look like.

It is assumed that there are $K$ essential attribute dimensions relevant to the birth/no birth decision. Each dimension represents an aspect of the prospective parent's life. Each level of each dimension corresponds to a distinct way that that aspect of the decision maker's life could turn out. For example, one dimension might be "Loneliness at age 65," with levels "High" and "Low," to make things simple. Another might be "Personal freedom at age 28," again with levels "High" and "Low." If the generic dimension is represented by $Y_{k}$, then the entire collection of possible outcomes of the decision might be representable as the Cartesian product $Y=\prod_{k=1}^{K} Y_{k}$. In a sense, each outcome vector $\left(y_{1 j_{1}}, y_{2 j_{2}}, \ldots, y_{K j_{K}}\right)$ in that collection might be seen as a distinct "life" for the decision maker which might be realized after the decision is made and executed.

From the SEU perspective, one of the critical elements of the decision maker's dilemma is that the likelihoods of particular vectors actually occurring differ, depending on whether the prospective parent chooses to have or not have a child. The other essential element is that the decision maker does not value all the vectors equally. The outcome vectors might be seen as partitioning the sample space of potential occurrences. If the 
decision maker accepts the SEU axioms, then it should be possible to find probability measures $P_{\mathrm{B}}$ and $P_{\mathrm{NB}}$ defined over partitions of the sample space encoding the decision maker's judgments of the chances of particular outcome vectors being realized if the decision maker were to, respectively, have or not have a child. Also, it should be possible to find a utility function $u$ defined over $Y$ which encodes the decision maker's preferences among the vectors as well as, implicitly, his or her attitude toward risk. Figure 3 highlights the constructs required by utility theory.

The implied probability measures and utility function have the special

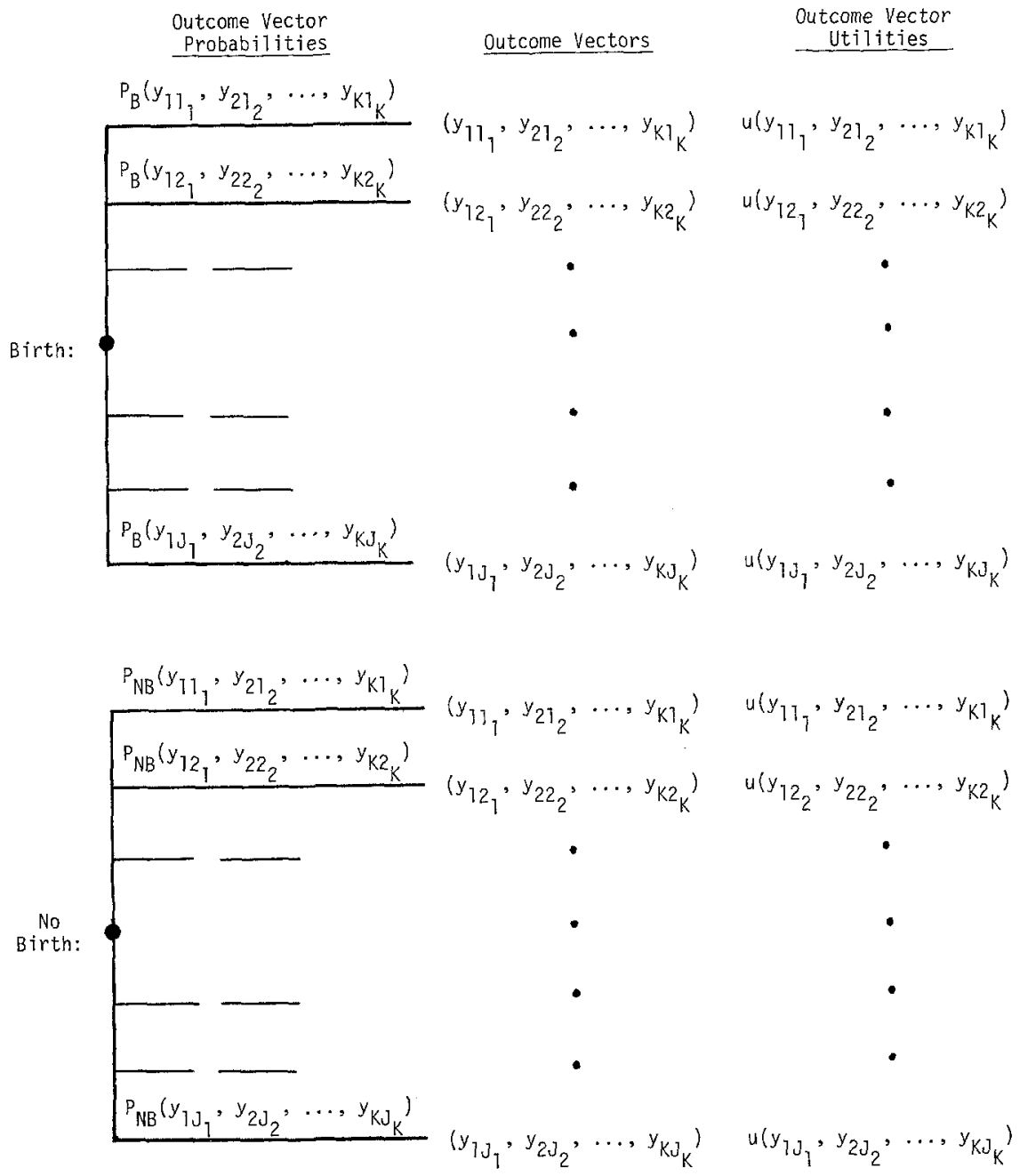

FIG. 3. Representations of the Birth and No Birth options, according to a standard utility theory analysis. 
property that the decision maker should choose Birth over No Birth only if $\mathrm{SEU}($ Birth) $\geqslant \operatorname{SEU}($ No Birth), where

$$
\operatorname{SEU}(\text { Birth })=\sum_{j_{k}} P_{\mathrm{B}}\left(y_{1 j_{1}}, y_{2 j_{2}}, \ldots, y_{K j_{K}}\right) u\left(y_{1 j_{1}}, y_{2 j_{2}}, \ldots, y_{K j_{K}}\right)
$$

and

SEU(No Birth $)=\sum_{j_{k}} P_{\mathrm{NB}}\left(y_{1_{1}}, y_{2 j_{2}}, \ldots, y_{K j_{K}}\right) u\left(y_{1 j_{1}}, y_{2 j_{2}}, \ldots, y_{K j_{K}}\right)$.

Otherwise, one or more of the SEU axioms might be violated. The summations in the above equations should be seen as generalized summation operators which would be integrals for continuous attribute dimensions.

In principle, the approach outlined above is simple. "All" the decision analyst has to do is elicit the probability measures and utility function from the client and apply formulas. In practice, the task is formidable indeed. Decision analysts worry about whether the decision maker has the intellectual capacity to meaningfully generate the required responses. For example, it is hard to even think about judging the likelihood of the following outcome vector, under the assumption that one has a child: ("Very lonely at age 65," "Well-off financially at age 32," "Teach family traditions"). Moreover, the number of distinct vectors in $Y=\prod_{k=1}^{K} Y_{k}$ for even a modest $K$ is likely to be extremely large. Thus, assigning utilities for all those vectors should be quite tedious and errorful. Provided that the decision maker agrees with certain principles, it can be shown (Keeney \& Raiffa, 1976) that the overall utility function $u$ can be expressed as a simple, e.g., additive, function of dimensional utility functions. Then the complexity of the vector utility assessment task is reduced considerably because one has to elicit only scalar utility functions. It might be noted that Keeney and Raiffa's (1976) techniques include hierarchical approaches similar in spirit to those favored by Beach $e t$ al.

Even the sorts of simplifications discussed by Keeney and Raiffa (1976) will be insufficient to permit one to operationalize feasibly the kind of EU maximization scheme described above in personal decision situations. Thus, additional simplifying assumptions are necessary. Hopefully, however, assumptions which do not do fundamental violence to the integrity of utility theory can be found.

Many of the implicit and explicit assumptions made by Beach et al. appear motivated by the need for simplification. As implied by the first of our suggested options for dealing with the formal problems of the Beach procedure, perhaps it can be shown that those assumptions are plausible 
and complement rather than conflict with utility theory. We are skeptical about this possibility for at least two reasons, however.

First, Beach et al.'s complementarity assumption would require a rigid relationship between probability measures $P_{\mathrm{B}}$ and $P_{\mathrm{NB}}$ in the standard SEU analysis. Specifically, it must always be the case that

$$
P_{\mathrm{NB}}\left(y_{1 j_{1}}, y_{2 j_{2}}, \ldots, y_{K j_{K}}\right)=1-P_{\mathrm{B}}\left(y_{1 j_{1}}, y_{2 j_{2}}, \ldots, y_{K j_{K}}\right) .
$$

It is doubtful that many decision makers' probability judgments would conform to this restriction.

A second reason for our skepticism is that Beach et al.'s formulation of the decision problem seems to demand two utility functions rather than just one, as in the traditional utility theory formulation. One utility function applies to the Birth option, the other to the No Birth option. Moreover, Beach et al.'s sign symmetry assumption specifies a one-toone relationship between those two utility functions. So, while for outcome vector $\left(y_{1 j_{1}}, y_{2 j_{2}}, \ldots, y_{K_{K}}\right)$ the standard analysis implies the existence of a single utility $u\left(y_{1 j_{1}}, y_{2 j_{2}}, \ldots, y_{K j_{K}}\right)$, the Beach approach requires two utilities, $u_{\mathrm{B}}\left(y_{1 j_{1}}, y_{2 j_{2}}, \ldots, y_{K j_{K}}\right)$ for the Birth option, and $u_{\mathrm{NB}}\left(y_{1 j_{1}}, y_{2 j_{2}}, \ldots, y_{K_{K}}\right)=-u_{\mathrm{B}}\left(y_{1 j_{1}}, y_{2 j_{2}}, \ldots, y_{K_{K}}\right)$ for the No Birth option. Substantively, the sign symmetry assumption says that the decision maker always values the same outcome differently, depending upon whether he or she does or does not have a child. For example, one would not feel the same about being rich at age 45 if one had the prospective child as one would feel if he or she did not have the child. We question the generality of such value patterns.

Although the basic idea behind the value patterns assumed by Beach $e t$ al. can be incorporated into a traditional utility theory framework, it requires a redefinition of the outcome vectors. Specifically, the $K$ dimensional vector $\left(y_{1 j_{1}}, y_{2 j_{2}}, \ldots, y_{K j_{K}}\right)$ must be replaced by a pair of $(K$ +1 )-dimensional vectors, (Child, $y_{1 j_{1}}, y_{2 j_{2}}, \ldots, y_{K j_{K}}$ ) and (No Child, $y_{1 j_{1}}$, $y_{2 j_{2}}, \ldots, y_{K j_{K}}$ ). The sign symmetry assumption would then say that $u$ (No Child, $\left.y_{1 j_{1}}, y_{2 j_{2}}, \ldots, y_{K j_{K}}\right)=-u$ (Child, $\left.y_{1 j_{1}}, y_{2 j_{2}}, \ldots, y_{\mathrm{Kj}_{K}}\right)$. The uniqueness theorem for utility theory says that, if $u$ is a satisfactory utility function, then so is $v=a u+b$, where $a>0$ (see, e.g., Luce \& Raiffa, 1957, p. 30). The sign symmetry assumption would also require that $v$ (No Child, $\left.y_{1 j_{1}}, y_{2 j_{2}}, \ldots, y_{K j_{K}}\right)=-v\left(\right.$ Child, $\left.y_{1 j_{1}}, y_{2 j_{2}}, \ldots, y_{K j_{K}}\right)$. It is easy to show that these conditions imply that $b=0$, i.e., $v=a u$, with $a>0$. Thus, ratio- rather than conventional interval-scale utility scaling is implied.

The use of positive and negative ratio-scaled utility values implies a sign dependence (Krantz et al., 1971, pp. 329-339) which seems consistent 
with the intentions of Beach et al. That is, not only is there a meaningful conceptual "zero outcome," but preferences among outcomes on one side of that zero outcome are in a particular sense mirror images of preferences among outcomes on the other side: "outcomes that would be gains if one were to have the child can be seen as losses if one were to not do so, and vice versa" (Beach et al., 1976, p. 102). But is it reasonable, for example, to expect a prospective parent to consider the outcome vector (Child, "Rich at age 45") a gain and the outcome vector (No Child, "Rich at age 45 ") a loss, or conversely?

\section{REFERENCES}

Beach L. R., Campbell, F. L., \& Townes, B. D. Subjective expected utility and the prediction of birth-planning decisions. Organizational Behavior and Human Performance, $1979,24,18-28$.

Beach, L. R., Townes, B. D., \& Campbell, F. L. The optional parenthood questionnaire: A guide to decision making about parenthood. Washington, D.C.: National Alliance for Optional Parenthood, 1978. (a)

Beach, L. R., Townes, B. D., \& Campbell, F. L. The clinician's manual for use with the optional parenthood questionnaire. Washington, D.C.: National Alliance for Optional Parenthood, 1978. (b)

Beach, L. R., Townes, B. D., Campbell, F. L., \& Keating, G. W. Developing and testing a decision aid for birth planning decisions. Organizational Behavior and Human Performance, 1976, 15, 99-116.

DeGroot, M. Optimal statistical decisions. New York: McGraw-Hill, 1970.

Gardiner, P. E., \& Edwards, W. Public values: Multiattribute-utility measurement for social decision making. In M. F. Kaplan \& S. Schwartz (Eds.), Human judgment and decision processes. New York: Academic Press, 1975.

Hershey, J. C., Kunreuther, H. C., \& Schoemaker, P. J. H. Sources of bias in assessment procedures for utility functions. Management Science, in press.

Herstein, I. H., \& Milnor, J. An axiomatic approach to measurable utility. Econometrica, $1953,21,291-297$.

Howard, R. A. Decision analysis in systems engineering. In R. F. Miles (Ed.), Systems concepts: lectures on contemporary approaches to systems. New York: Wiley, 1973.

Keeney, R. L., \& Raiffa, H. Decisions with multiple objectives: Preferences and value tradeoffs. New York: Wiley, 1976.

Krantz, D. H., Luce, R. D., Suppes, P., \& Tversky, A. Foundations of measurement, Vol. 1. New York: Academic Press, 1971.

Luce, R. D., \& Raiffa, H. Games and decisions. New York: Wiley, 1957.

Morris, W. T. Decision analysis. Columbus, Ohio: Grid, 1977.

Pratt, J. W. Risk aversion in the small and in the large. Econometrica, 1964, 32, 122-136.

Savage, L. J. The foundations of statistics. New York: Wiley, 1954.

Schlaifer, R. Analysis of decisions under uncertainty. New York: McGraw-Hill, 1969.

von Neumann, J., \& Morgenstern, O. Theory of games and economic behavior. Princeton, N.J.: Princeton Univ. Press, 1944.

Weinstein, M. C., \& Fineberg, H. V. Clinical decision analysis. Philadelphia: Saunders, 1980.

Winkler, R. L. An introduction to Bayesian inference and decision. New York: Holt, Rinehart \& Winston, 1972. 
Winkler, R. L., \& Murphy, A. H. Experiments in the laboratory and the real world. Organizational Behavior and Human Performance, 1973, 10, 252-270.

Wood, R. J., Campbell, F. L., Townes, B. D., \& Beach, L. R. Birth planning decisions. American Journal of Public Health, 1977, 67, 563-565.

\section{REFERENCE NOTES}

1. Selvidge, J. E. Methodology for the retrospective evaluation of decision analysis (Tech. Rep. No. 76-13). McLean, Va.: Decisions and Designs, 1976.

2. Yates, J. F., \& Carlson, B. W. Experiments related to representation theory. Invited paper presented at the Eighth Conference on Subjective Probability, Utility, and Decision Making, Budapest, Hungary, August 1981.

Received: November 6, 1981. 